\title{
One Fusion Fault Diagnosis Method of Grey Entropy Relation and Fuzzy Cluster
}

\author{
Yanqin Su ${ }^{1, *}$, Guangyi Zhang ${ }^{2}$ and Libin Yang ${ }^{3}$ \\ ${ }^{1}$ Department of Control Engineering, Naval Aeronautical and Astronautical University, Yantai Shandong, China \\ ${ }^{2}$ Department of Science Research, Naval Aeronautical and Astronautical University, Yantai Shandong, China \\ ${ }^{3}$ Department of weapon science and engineering, Naval Aeronautical and Astronautical University, Yantai Shandong, China \\ *Corresponding author
}

\begin{abstract}
There are the problem that it must be given the reference standard sequence ahead according to the experience or statistics method in the classical grey entropy relation for fault diagnosis. But it is often lack of objectivity and high accuracy degree. The paper introduced one advanced Fuzzy C-Mean cluster to get the reference standard sequence, which not only mended the above shortage, but also was applied to some equipment to verify its validity and superiority.
\end{abstract}

Keywords-grey entropy relationship; fuzzy c-mean cluster; fault diagnosis

\section{INTRODUCTION}

In the equipment fault diagnosis, because the test equipment has some limit, the fault data is often grey of part known and part unknown. The Grey System Theory has some advantage to process the data, in which the grey relationship method is fault mode recognition through calculating the relationship degree between the detected mode or comparison sequence and the fault mode or reference sequence [1]. Grey entropy relationship method uses the entropy to express the relationship degree, and takes full advantage of the individuality information to realize the entire approach, so it is more scientific and reasonable [2].

However, it needs a reference sequence beforehand in the grey entropy relationship fault diagnosis method, which is often given by the experience or statistics [3]. In order to increase the objectivity and veracity, the Fuzzy C-Mean (FCM) cluster is introduced to get the $C$ optimal cluster center as $C$ grey category for generating the reference sequence. The fusion method based on FCM and Grey entropy relationship is applied to some example fault diagnosis to verify its effectiveness and superior.

\section{GREY ENTROPY RELATIONSHIP METHOD AND ITS DISADVANTAGE}

The part information sequence is named as grey connotation sequence [4]. Order grey connotation sequence $X$, $x_{i}(k) \in X \quad, \quad i=1,2, \cdots, m, k=1,2, \cdots n$. The grey relationship coefficient $\gamma(k)$ and the grey relationship degree $\gamma_{i}$ between the comparison sequence $X_{i}$ and the reference sequence ${ }^{X_{0}}$ at the $k$ point is defined respectively as

$$
\begin{gathered}
\gamma(k)=\frac{\min _{i} \min _{k}\left|x_{0}(k)-x_{i}(k)\right|+\rho \max _{i} \max _{k}\left|x_{0}(k)-x_{i}(k)\right|}{\left|x_{0}(k)-x_{i}(k)\right|+\rho \max _{i} \max _{k}\left|x_{0}(k)-x_{i}(k)\right|} \\
\gamma_{i}=\frac{1}{n} \sum_{k=1}^{n} \gamma(k)
\end{gathered}
$$

In which, $\rho$ is differentiated coefficient, and $\rho=0.5$ generally.

The grey entropy of sequence $X=\left(x_{1}, x_{2}, \cdots, x_{n}\right)$ is defined as[4]

$$
H=-\sum_{j=1}^{n}\left[x_{j} \bullet \ln x_{j}\right]
$$

In which, $H_{\max }=\ln n$.

Thus, the grey relationship entropy is

In which,

$$
\begin{aligned}
& H_{i}=-\sum_{k=1}^{n}\left[p_{i}(k) \bullet \ln p_{i}(k)\right] \\
& p_{i}(k)=\frac{\gamma\left[x_{0}(k), x_{i}(k)\right]}{\sum_{k=1}^{n} \gamma\left[x_{0}(k), x_{i}(k)\right]}
\end{aligned}
$$

$p_{i}(k)$ is named as the relationship density value between the comparison sequence ${ }^{X_{i}}$ and the reference sequence ${ }^{X_{0}}$ at the $k$ point. The mapping value $p_{i}(k)$ fulfills $p_{i}(k) \geq 0$ 且 $\sum_{k=1}^{n} p_{i}(k)=1$ 
The grey entropy relationship degree is defined as

$$
E_{i}=\frac{H_{i}}{H_{\max }}
$$

According to the entropy gain theorem, the more grey entropy relationship $E_{i}$, the lager the relationship degree between the comparison sequence $x_{i}$ and the reference sequence $X_{0}$

The steps of the classical grey entropy relationship fault diagnosis method are shown in the FIGURE I.

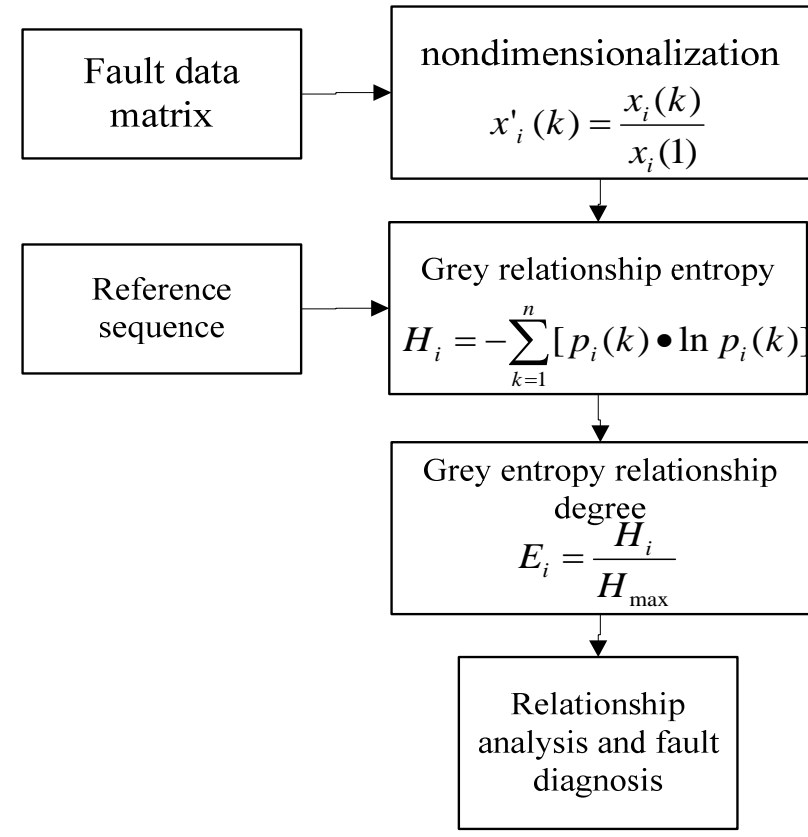

FIGURE I. THE STEPS OF THE CLASSICAL GREY ENTROPY RELATIONSHIP FAULT DIAGNOSIS METHOD

It needs a reference sequence beforehand in the grey entropy relationship fault diagnosis method, which is often given by the experience or statistics.

\section{ONE ADVANCED FUSION FAULT DIAGNOSIS METHOD BASED ON FUZZY C-MEAN CLUSTER AND GREY ENTROPY RELATIONSHIP}

In the paper, the Fuzzy C-Mean (FCM) cluster is introduced to get the $C$ optimal cluster center as $C$ grey category for generating the reference sequence. FCM is one cluster method based on target function, which considers every data point is belong to each difference category as the fixed subordinate degree. The fuzzy procession can reflect the real distribution of the data, which is an objective and effective method of confirming the reference standard sequence. Meanwhile, the FCM cluster based on extreme difference specification can used to nondimensionalize[6]. The steps are summarized as bellow:

\section{A. Collect The Fault Data}

According to the evaluation target, the evaluation index system is established, and then evaluation data is collected to set up the data matrix.

$$
\left(X_{0}, X_{1}, \cdots, X_{m}\right)=\left[\begin{array}{llll}
X_{0}(1) & X_{1}(1) & \cdots & X_{m_{1}}(1) \\
X_{0}(2) & X_{1}(2) & \cdots & X_{m_{1}}(2) \\
\cdots & & & \\
X_{0}(n) & X_{1}(n) & \cdots & X_{m_{1}}(n)
\end{array}\right]
$$

\section{B. Extreme Difference Specification}

$\mathrm{T}$ Extreme difference specification is applied in the nondimensionalization, and the comparison sequence is introduced in the specification formula

$$
\begin{aligned}
& x_{i j}=\frac{x_{i j}-x_{j \min }}{x_{j \max }-x_{j \min }},(i=1,2, \cdots, n ; j=1,2, \cdots, m) \\
& \text { In } \quad \text { which, } \quad x_{j \max }=\max \left\{x_{1 j}, x_{2 j}, \cdots, x_{n j}\right\}, \\
& x_{j \min }=\min \left\{x_{1 j}, x_{2 j}, \cdots, x_{n j}\right\} . \\
& \text { C. Confirm The Referenced Standard Sequence Based on } \\
& \text { FCM }
\end{aligned}
$$

\section{Confirm The Referenced Standard Sequence Based on FCM}

Through FCM cluster to get the $C$ optimal cluster center of the reference sequence and every cluster center can be recorded as $V(k)=\left[v_{1}(k), v_{2}(k), \cdots, v_{n}(k)\right]^{T}, k(k=1,2, \cdots, l)$.

(1) The sample set is divided as $D=\left(\left\{d_{1}, d_{2}, \cdots, d_{c}\right\}\right)$, $2 \leq c \leq n$ categories, and the matrix $V$ generated by the $C$ cluster center vector

$$
V=\left[\begin{array}{c}
V_{1} \\
V_{2} \\
\cdots \\
V_{C}
\end{array}\right]\left[\begin{array}{cccc}
v_{11} & v_{12} & \cdots & v_{1 m} \\
v_{21} & v_{22} & \cdots & v_{2 m} \\
\cdots & \cdots & \cdots & \cdots \\
v_{C 1} & v_{C 2} & \cdots & v_{C m}
\end{array}\right]
$$

Initialization. Given the needed cluster category number $C, 2 \leq C \leq n, n$ is the sample number, in which the category cannot exceed the sample number nor equal to 1 . Confirm the fuzzy factor $m(1 \leq m \leq \infty)$ and the iteration threshold $\varepsilon$, the maximum iteration times $N$, the classification matrix $W$; 
(2) The sample mean is used to calculate the cluster center $v_{1}^{(0)}, v_{2}^{(0)}, \cdots, v_{C}^{(0)}$, and the cluster center matrix is $V=\left(v_{1}^{(0)}, v_{2}^{(0)}, \cdots, v_{C}^{(0)}\right)^{T}$;

(3) Calculate the fuzzy classification matrix, and iteration update

$$
w_{i j}=\left[\sum_{k=1}^{C}\left(\frac{\left\|x_{j}-v_{i}\right\|^{2}}{\left\|x_{j}-v_{k}\right\|^{2}}\right)^{\frac{1}{m-1}}\right]^{-1}, 1 \leq i \leq C, 1 \leq j \leq n
$$

(4) Calculate the cluster center, and iteration update

$$
V_{i}(k+1)=\frac{\sum_{i=1}^{n} w_{i j}^{2}(k) x_{i}}{\sum_{i=1}^{n} w_{i j}^{2}(k)}
$$

(5) If target function

$$
J_{m}^{\prime}(U, W, V)=\sum_{j=1}^{n} \sum_{i=1}^{C} w_{i j}^{m}\left\|x_{j}-v_{i}\right\|^{2}
$$

achieve the threshold $\varepsilon$ or the maximum iteration times $N$, end iteration, or jump to step 2. Lastly, gain the cluster center $C$ and their subordinate degree divide matrix $W$.

\section{Fault Diagnosis}

Calculate the grey relationship entropy $H_{i}^{\prime}=-\sum_{k=1}^{n}\left[p_{i}^{\prime}(k) \bullet \ln p_{i}^{\prime}(k)\right]$ relative to the cluster center $C$, in which, $p_{i}^{\prime}(k)=\frac{\gamma\left[x_{0}^{\prime}(k), x_{i}^{\prime}(k)\right]}{\sum_{k=1}^{n} \gamma\left[x_{0}^{\prime}(k), x_{i}^{\prime}(k)\right]}$. The relationship analysis of the new grey entropy relationship degree $E_{i}^{\prime}=\frac{H_{i}^{\prime}}{H_{\text {max }}^{\prime}}$ is calculated to judge the fault mode [7].

\section{APPLICATION EXAMPLE AND ANALYSIS}

The paper chooses the aviation radio equipment as the diagnosis object, and the fault diagnosis locates at the chip or module level. Table I gives the fault phenomenon of "the radio station is uncontrolled", in which the fault omen is expressed as condition attribution " $C$ ", $C=\left\{+5 \mathrm{~V}\right.$ voltage $C_{1}$, UUT receiver voltage $C_{2}$, UUT transmitter voltage $C_{3}, 1553 \mathrm{~B}$ bus $\left.C_{4}\right\}$, decision attribution $D=\left\{d_{1}, d_{2}\right\}$, “ $d_{1}$ ”means fault front-panel, “ $d_{2}$ ” means fault host PC.

TABLE I. FAULT DATA

\begin{tabular}{|c|c|c|c|c|c|}
\hline No. & $\boldsymbol{C}_{\mathbf{1}}$ & $\boldsymbol{C}_{\mathbf{2}}$ & $\boldsymbol{C}_{\boldsymbol{3}}$ & $\boldsymbol{C}_{\mathbf{4}}$ & $\boldsymbol{D}$ \\
\hline$(1)$ & 5.8 & 36.0 & 55.0 & 40 & $D_{1}$ \\
\hline$(2)$ & 6.7 & 45.0 & 35.5 & 50 & $D_{1}$ \\
\hline$(3)$ & 5.3 & 35.5 & 44.5 & 50 & $D_{1}$ \\
\hline$(4)$ & 7.5 & 49.0 & 50.5 & 55 & $D_{1}$ \\
\hline$(5)$ & 5.4 & 40.5 & 40.0 & 45 & $D_{1}$ \\
\hline$(6)$ & 4.9 & 27.0 & 27.5 & 210 & $D_{2}$ \\
\hline$(7)$ & 5.0 & 27.5 & 27.5 & 270 & $D_{2}$ \\
\hline$(8)$ & 5.0 & 27.5 & 27.0 & 350 & $D_{2}$ \\
\hline$(9)$ & 5.1 & 28.0 & 28.0 & 330 & $D_{2}$ \\
\hline$(10)$ & 5.0 & 28.0 & 28.5 & 300 & $D_{2}$ \\
\hline$(11)$ & 5.0 & 30.5 & 28.5 & 130 & $D_{1}$ \\
\hline$(12)$ & 5.1 & 29.5 & 27.5 & 410 & $D_{2}$ \\
\hline
\end{tabular}

Firstly, the front 10 group fault data is nondimensionalized of extreme difference specification, and the results is shown as Table II.

TABLE II. THE NONDIMENSIONALIZED RESULTS

\begin{tabular}{|c|c|c|c|c|c|}
\hline No. & $\boldsymbol{C}_{\mathbf{1}}$ & $\boldsymbol{C}_{\mathbf{2}}$ & $\boldsymbol{C}_{\mathbf{3}}$ & $\boldsymbol{C}_{\mathbf{4}}$ & $\boldsymbol{D}$ \\
\hline$(1)$ & 0.34615 & 0.40909 & 1 & 0 & $D_{1}$ \\
\hline$(2)$ & 0.69231 & 0.81818 & 0.30357 & 0.032258 & $D_{1}$ \\
\hline$(3)$ & 0.15385 & 0.38636 & 0.625 & 0.032258 & $D_{1}$ \\
\hline$(4)$ & 1 & 1 & 0.83929 & 0.048387 & $D_{1}$ \\
\hline$(5)$ & 0.19231 & 0.61364 & 0.46429 & 0.016129 & $D_{1}$ \\
\hline$(6)$ & 0 & 0 & 0.017857 & 0.54839 & $D_{2}$ \\
\hline$(7)$ & 0.038462 & 0.022727 & 0.017857 & 0.74194 & $D_{2}$ \\
\hline$(8)$ & 0.038462 & 0.022727 & 0 & 1 & $D_{2}$ \\
\hline$(9)$ & 0.076923 & 0.045455 & 0.035714 & 0.93548 & $D_{2}$ \\
\hline$(10)$ & 0.038462 & 0.045455 & 0.053571 & 0.83871 & $D_{2}$ \\
\hline
\end{tabular}

Then, because the fault module has 2 categories, the cluster number is set to $C=2$. And then, the FCM cluster is applied to get the reference standard sequence as Table III.

TABLE III. THE REFERENCE STANDARD SEQUENCE

\begin{tabular}{|c|c|c|c|c|c|}
\hline No. & $\boldsymbol{C}_{\mathbf{1}}$ & $\boldsymbol{C}_{\mathbf{2}}$ & $\boldsymbol{C}_{\mathbf{3}}$ & $\boldsymbol{C}_{\mathbf{4}}$ & $\boldsymbol{D}$ \\
\hline$(1)$ & 0.47366 & 0.64442 & 0.64349 & 0.025835 & $D_{1}$ \\
\hline$(2)$ & 0.044883 & 0.035652 & 0.033412 & 0.80642 & $D_{2}$ \\
\hline
\end{tabular}

After then, the (11) and (12) group fault data is applied FCM cluster to get cluster center, which is calculated their grey entropy relationship degree as Table IV.

TABLE IV. THE GREY ENTROPY RELATIONSHIP DEGREE

\begin{tabular}{|c|c|c|}
\hline No. & $X_{01}$ & $X_{02}$ \\
\hline$(11)$ & 0.25176 & 0.22273 \\
\hline$(12)$ & 0.22469 & 0.26102 \\
\hline
\end{tabular}


According to grey entropy relationship degree of the (11) and the (12) group fault data, the fault mode is decided, and the fault mode is got as $D_{1}$ and $D_{2}$ respectively, which is verified their accordance with the test data. So the method is effective, which is settled the base to the next fault diagnosis application. The FCM is used to get the reference standard sequence, which use the test data themselves and need not any subjectivity factor, the accuracy is higher. It has some diagnosis advantage of the situation of the fuzzy fault omen and the inconspicuous fault boundary.

\section{CONCLUSION}

Through studying the disadvantage of the classical grey entropy relationship, the reference standard sequence fault diagnosis method based on FCM is given, and the example is verified the effectiveness, which provides a new way to study the grey theory fault diagnosis more objectively.

\section{REFERENCES}

[1] Liu Sifeng, Xie Naiming. Grey system Theory and Application [M]. Beijing: Science Press, 2008.

[2] Huang Liang, Hou Jianjun, Liu Ying, Li Jihong. Algorithm of selecting optimal test point for analog circuit based on grey relational entropy [J]. Journal of Electronic Measurement and Instrument, 2009,9(23): 27-33.

[3] Cao Jian, Fan Jinmin, Ding Jiafeng, Sheng Chi. Fault Diagnosis of Transformer Using an Improved Grey Entropy Relation Algorithm [J]. Proceedings of the CSU-EPSA, 2009, 4(21):25-30.

[4] Deng Julong. Grey System Theory [M]. Wuhan: CCNU press, 2008.

[5] Chen Qi, Qi Xiaohui. Fault Diagnosis of Engines Based on Grey Correlative Analysis [J]. Journal of Tianjin University of Commerce, 2007, 6(27):6-8.

[6] Meng Xiaoyao, Han Xinjie. The Fuzzy C-Means Cluster Algorithm and Its Application in the Fault Diagnosis of Ships [J]. Shipbuilding of China, 2007,4(48): 98-103.

[7] Deng Honggui, Luo An, Cao Jian. Application of Grey Relative Degree to Fault Diagnosis of Transformer [J]. Control Engineering of China, 2005,1 (12):25-28. 